\title{
More Style, Less Work: Card-style Data Decrease Risk-limiting Audit Sample Sizes
}

\author{
AMANDA K. GLAZER, JACOB V. SPERTUS, and PHILIP B. STARK, Department of Statistics, Uni- \\ versity of California, Berkeley
}

U.S. elections rely heavily on computers such as voter registration databases, electronic pollbooks, voting machines, scanners, tabulators, and results reporting websites. These introduce digital threats to election outcomes. Risk-limiting audits (RLAs) mitigate threats to some of these systems by manually inspecting random samples of ballot cards. RLAs have a large chance of correcting wrong outcomes (by conducting a full manual tabulation of a trustworthy record of the votes), but can save labor when reported outcomes are correct. This efficiency is eroded when sampling cannot be targeted to ballot cards that contain the contest(s) under audit. If the sample is drawn from all cast cards, then RLA sample sizes scale like the reciprocal of the fraction of ballot cards that contain the contest(s) under audit. That fraction shrinks as the number of cards per ballot grows (i.e., when elections contain more contests) and as the fraction of ballots that contain the contest decreases (i.e., when a smaller percentage of voters are eligible to vote in the contest). States that conduct RLAs of contests on multi-card ballots or RLAs of small contests can dramatically reduce sample sizes by using information about which ballot cards contain which contests-by keeping track of card-style data (CSD). For instance, CSD reduce the expected number of draws needed to audit a single countywide contest on a 4 -card ballot by $75 \%$. Similarly, CSD reduce the expected number of draws by $95 \%$ or more for an audit of two contests with the same margin on a 4-card ballot if one contest is on every ballot and the other is on $10 \%$ of ballots. In realistic examples, the savings can be several orders of magnitude.

CCS Concepts: • Applied computing $\rightarrow$ Voting/election technologies; Computers in other domains; Computing in government;

Additional Key Words and Phrases: Elections, audits, risk-limiting, ballots, card-style, sampling

ACM Reference format:

Amanda K. Glazer, Jacob V. Spertus, and Philip B. Stark. 2021. More Style, Less Work: Card-style Data Decrease Risk-limiting Audit Sample Sizes. Digit. Threat.: Res. Pract. 2, 4, Article 32 (October 2021), 15 pages.

https://doi.org/10.1145/3457907

Style is a way to say who you are without having to speak.

\section{INTRODUCTION}

- Rachel Zoe

The principle of evidence-based elections is that elections should provide convincing evidence that the reported winners really won [20]. Evidence-based elections require a trustworthy record of the votes. Generally, that means hand-marked paper ballots (with an accessible marking option for voters who require accommodations) kept demonstrably secure throughout the canvass $[1,2]$. Crucially, the reported results must be checked against that trustworthy paper trail. Risk-limiting audits (RLAs) provide a rigorous way to perform that check so that

Jacob Spertus' work was supported by a National Science Foundation (NSF) Graduate Research Fellowship (DGE 1752814). Amanda Glazer's work was supported by an NSF grant (DMS RTG \#1745640).

Author's address: A. K. Glazer, J. V. Spertus, and P. B. Stark, Department of Statistics, University of California, Berkeley; emails: amandaglazer@berkeley.edu, jakespertus@berkeley.edu, stark@stat.berkeley.edu.

Permission to make digital or hard copies of part or all of this work for personal or classroom use is granted without fee provided that copies are not made or distributed for profit or commercial advantage and that copies bear this notice and the full citation on the first page. Copyrights for third-party components of this work must be honored. For all other uses, contact the owner/author(s).

(c) 2021 Copyright held by the owner/author(s).

$2576-5337 / 2021 / 10-$ ART32 $\$ 15.00$

https://doi.org/10.1145/3457907 
there is a large chance of correcting the reported outcome if it is wrong. The probability that an RLA does not correct the reported outcome if the reported outcome is wrong is less than the risk limit [10, 16].

U.S. elections generally include several to dozens of contests that widely vary in size: Each voter is eligible to vote in a subset of those contests. For example, a voter may be eligible to vote for president, senators, governor, representatives, mayor, judges, school board seats, local tax measures, and so on. Because ballots often contain so many contests, they generally comprise more than one card or sheet or page, each containing some of the contests.

The term ballot style generally refers to the set of contests on a given voter's ballot. (Ballot style can also encode precinct information, i.e., even if voters in two different precincts are eligible to vote in the same set of contests, ballots for the two precincts are considered to be of two different styles.) Here, we use card style to refer to the set of contests on a given ballot card, and CSD to refer to card-style data for an election.

RLAs generally involve inspecting a random sample of ballot cards. States that perform RLAs have so far drawn the sample of ballot cards to inspect from all the cards cast in the election, without regard for the contests those cards contain. When a contest under audit appears on only a small fraction of ballot cards, this can make the audit unnecessarily costly. Especially when the margin ${ }^{1}$ is small, an RLA based on a sample drawn from all ballot cards requires manually inspecting far more cards than an RLA that only samples cards that contain the contest. Targeting the sample to just those cards is possible if card-style data are available, that is, a listing of the contests each cast card contains.

A similar issue arises for large contests-even jurisdiction-wide contests-when ballots consist of multiple cards: If the sample can be drawn just from cards that contain those contests, then a much smaller sample may suffice to confirm the outcome than if the sample is drawn indiscriminately from all cast cards [9]. As a rule of thumb, if a contest is on a fraction $f$ of ballot cards cast in the contest, then the sample size required to confirm the contest outcome will be roughly $1 / f$ times larger than if the sample can be drawn just from cards that contain the contest.

Here, we show that CSD-i.e., keeping track of which cards contain which contests-can reduce audit sample sizes by orders of magnitude in typical elections. Section 2 provides additional context and defines key terms. Section 3 examines the simplest case with a ballot-level comparison audit: auditing two contests in an election with a one-card ballot. One contest is on every card; the other is on only some of the cards. Section 4 considers auditing two contests in an election with multi-card ballots. Section 5 extends our analysis to ballot-polling audits. Section 6 presents case studies of hypothetical audits in two California counties of different sizes. Section 7 sketches how to implement an audit that takes advantage of CSD using consistent sampling. Section 8 presents conclusions and recommendations.

\section{BACKGROUND}

\subsection{Ballots, Cards, Ballot Manifests, and Card Styles}

A ballot is what the voter receives and casts; a ballot card is an individual page of a ballot. In the U.S., ballots often consist of more than one card. The ballot cards that together comprise a ballot generally do not stay together once they are cast. RLAs generally draw ballot cards at random-not "whole" ballots.

To conduct an RLA, an upper bound on the number of validly cast ballot cards must be known before the audit begins. The bound could come from manually keeping track of the paper, or from other information available to the election official, such as the number of voters eligible to vote in each contest, the number of pollbook signatures, or the number of ballots sent to polling places, mailed to voters, and returned by voters [3].

RLAs generally rely on ballot manifests to draw a random sample of ballot cards. A ballot manifest describes how the physical ballot cards are stored. It is the sampling frame for the audit. This article explains how it can

\footnotetext{
${ }^{1}$ Technically, the diluted margin [17] drives sample sizes for ballot-level comparison audits, as described below. The diluted margin is the margin in votes divided by the total number of cards in the population from which the sample is drawn. It is generally less than the conventional margin, which is the margin in votes divided by the number of valid votes in the contest, excluding undervotes and overvotes.
}

Digital Threats: Research and Practice, Vol. 2, No. 4, Article 32. Publication date: October 2021. 


\begin{tabular}{|l|l|l|l|l|l|}
\hline Cart & Tray & Position in Tray & Governor & Mayor of Irvine & $\ldots$ \\
\hline 1 & 4 & 96 & Yes & No & $\ldots$ \\
\hline 5 & 1 & 12 & Yes & No & $\ldots$ \\
\hline 2 & 2 & 72 & Yes & No & $\ldots$ \\
\hline$\ldots$ & $\ldots$ & $\ldots$ & $\ldots$ & $\ldots$ & $\ldots$ \\
\hline 3 & 5 & 50 & Yes & Yes & $\ldots$ \\
\hline
\end{tabular}

Fig. 1. A hypothetical example of CSD for an election in Orange County California with one-card ballots. Ballot cards are uniquely identified by their position (cart, tray, position in tray). CSD further identify contests that each ballot card is supposed to contain (truncated to two contests here), appearing as columns. Here we display the records for the countywide Governor's race and the race for Mayor of Irvine (a city within Orange County). More storage-efficient CSD might associate an unstructured list to each ballot card with numeric identifiers of the contests it contains (e.g., $\{1,4,10,12\})$. The public should be able to check that there are $N$ lines in the CSD, where $N$ is the number of ballots (and ballot cards) cast in the county. If $N_{S}$ ballots were cast in contest $S$, then there should be $N_{S}$ lines with "yes" in the column corresponding to contest $S$ in the CSD.

\begin{tabular}{|l|l|l|l|l|l|}
\hline Cart & Tray & Position in Tray & Governor & Mayor of Irvine & $\ldots$ \\
\hline 2 & 6 & 3 & No & Yes & $\ldots$ \\
\hline 5 & 1 & 12 & No & No & $\ldots$ \\
\hline 2 & 5 & 64 & Yes & No & $\ldots$ \\
\hline$\ldots$ & $\ldots$ & $\ldots$ & $\ldots$ & $\ldots$ & $\ldots$ \\
\hline 1 & 2 & 8 & Yes & No & $\ldots$ \\
\hline
\end{tabular}

Fig. 2. An example of CSD for a hypothetical election in Orange County with multi-card ballots. The Governor's race and the race for Mayor of Irvine appear on different cards. Because neither contest can appear on a card in this example (e.g., line 2), there must be at least three card styles.

be beneficial to augment the ballot manifest with information about the style of each card, i.e., the particular contests the card contains-CSD. Until recently, ${ }^{2}$ CSD have not been used in RLAs. Figures 1 and 2, respectively, display examples of CSD for single-card and multi-card ballots.

There are two principal ways to generate CSD: (1) physically sort the ballot cards according to the contests they contain, or (2) rely on the voting system for that information-even though it might not be accurate. Precinctbased voting partially sorts ballot cards: If every voter in the precinct is eligible to vote in the same contest(s) and the ballot has only one card, then each precinct's ballot cards have a single style. Knowing which precinct a bundle of ballot cards came from then tells us the contests on each card. This does not work for multi-card ballots. Some jurisdictions sort vote-by-mail ballots by precinct before scanning, which also partially sorts the cards. Vote centers make sorting ballots more difficult, because each center receives ballot styles from more than one precinct, and ballots cast in vote centers generally are not sorted before they are scanned.

Some modern vote tabulation systems record a cast-vote record (CVR) (a record of how the voting system interpreted the selections on the ballot card) for each ballot card in a way that allows the corresponding physical card to be identified and retrieved, and vice versa. Such systems are amenable to efficient RLAs and they also contain (possibly inaccurate) CSD: The contests on a card can be inferred from its CVR, as long as the CVR encodes "no selection" for contests in which the voter did not express a preference, according to the voting system. CSD derived from CVRs rely on the voting system, so they could be wrong: CSD might show that a card contains a contest it does not contain, or vice versa. Such errors can be accounted for rigorously in the audit using the "manifest phantoms to evil zombies" approach $[3,19]$, described below. The same approach can accommodate errors in CSD uncovered in auditing manually or machine-sorted ballot cards.

\footnotetext{
${ }^{2}$ In November 2019, a pilot RLA was conducted in San Francisco that used CSD [5].
} 
Because physically sorting cards is expensive, we expect that CSD generally will not be available unless the jurisdiction has a voting system that can produce CVRs linked to physical ballots. If such CVRs are available, then ballot-level comparison RLAs are possible. Such RLAs are especially efficient, so we emphasize them below.

\subsection{Ballot-polling and Ballot-level Comparison Audits}

There are two common approaches to auditing: comparison and ballot-polling [10] (there are also audits that combine the two approaches; see, e.g., Ottoboni et al. [13], Stark [19]). Comparison audits involve comparing manual tabulation of physically identifiable sets of ballot cards to the machine tabulation of the same ballot cards. The efficiency of comparison audits increases as the size of the sets decreases. The most efficient comparison audits compare human interpretation of individual ballot cards to the machine interpretation of individual ballot cards, CVRs. Such audits are called ballot-level comparison audits (in contrast to batch-level comparison audits, which compare the manual and electronic tabulation of batches of ballots, such as ballots cast in person in a particular precinct). Ballot-level comparison audits are possible only if the voting system produces CVRs that can be linked to the corresponding physical ballot card. ${ }^{3}$ Legacy voting systems generally do not, but many newer systems do.

Ballot-polling audits check the outcome but do not check or rely on the machine tabulation. They do not require CVRs or machine subtotals: all they require is paper ballots, organized well enough to sample cards at random.

Here, we focus on ballot-level comparison audits but we briefly address ballot-polling audits. We do not address batch-level comparison audits.

Ballot-level comparison audits and ballot-polling audits sample individual physical ballot cards. We refer to the act of sampling a single ballot card as a draw. Retrieving and inspecting ballot cards is labor intensive, so when the reported outcome is correct we want to minimize the number of draws (i.e., the sample size). We show below that knowing which cards contain which contests can dramatically reduce the number of draws required to confirm correct outcomes. (When the outcome is incorrect, we want the audit to inspect every ballot, to determine the correct outcome.)

\subsection{Super-simple Simultaneous Single-Ballot RLAs}

In this article we use the super-simple simultaneous single-ballot (S4) RLA of Stark [17] to illustrate workloads. Although S4 is not the most efficient RLA method, in part because it relies on sampling with replacement, it allows simple workload computations without the need for simulation. Moreover, if the sample size is small relative to the number of ballots cast and the number of observed discrepancies is small, the method is close to the best known. (If the sample size is an appreciable fraction of the population, then other methods can be far more efficient. See, e.g., Reference [19].) We expect that the savings in workload afforded by CSD will be substantial for all RLA methods.

The number of draws S4 needs to confirm results depends on the diluted margin and the number and nature of discrepancies the sample uncovers. ${ }^{4}$ The initial sample size can be written as a constant (denoted $\left.\rho\right)^{5}$ divided by the "diluted margin." With CSD, there are two relevant "diluted margins," as we shall see. The partially diluted margin is the margin in votes divided by the number of cards that contain the contest, including cards with undervotes or no valid vote in the contest. This differs from how margins are often reported, where the denominator

\footnotetext{
${ }^{3}$ There are also transitive ballot-level comparison audits, which involve re-scanning the ballot cards using an unofficial system.

${ }^{4}$ The S4 method has one tuning parameter, $\gamma$, which does not affect the risk limit but does affect the workload. To estimate the final workload, we assume that some fraction of the ballots in the sample will reveal 1-vote overstatements (errors that inflated a reported winner's margin over a reported loser by 1 vote). See Stark [17].

${ }^{5}$ In general, $\rho=-\log (\alpha) /\left[\frac{1}{2 \gamma}+\lambda \log \left(1-\frac{1}{2 \gamma}\right)\right]$, where $\gamma$ is an error inflation factor and $\lambda$ is the anticipated rate of one-vote overstatements in the initial sample as a percentage of the diluted margin [17]. We define $\gamma$ and $\lambda$ as in https:/www.stat.berkeley.edu/ stark/Vote/auditTools. htm.
}

Digital Threats: Research and Practice, Vol. 2, No. 4, Article 32. Publication date: October 2021. 
is only the valid votes in the contest, not the number of cards cast in the contest. The fully diluted margin is the margin in votes divided by the number of cards in the population of cards from which the audit sample is drawn. When the sample is drawn only from cards that contain the contest, the partially diluted margin and the fully diluted margin are equal; otherwise, the fully diluted margin is smaller. If CSD are unavailable, then the number of cards in that population is the number of cards cast in the jurisdiction. If CSD are available, then the number of cards in the population can be reduced to the number of cards that contain the contest. The availability of CSD drives the sample size through the difference between the partially and fully diluted margins.

\section{ONE-CARD BALLOTS}

Consider an election with a one-card ballot. We want to audit two contests: a jurisdiction-wide contest $B$ (for "big") listed on every card and a smaller contest $S$ (for "small") listed on some of the cards. For example, $B$ might be a countywide contest such as sheriff and $S$ might be a mayoral race.

There are $N$ ballots cast in the jurisdiction, of which $N_{B}=N$ contain contest $B$ and $N_{S}=p N<N$ contain contest $S$, where $p \in(0,1){ }^{6}$

The reported margin of contest $B$ is $M_{B}$ votes and the reported margin of contest $S$ is $M_{S}$ votes. Let $m_{B} \equiv M_{b} / N_{B}$ and $m_{S} \equiv M_{S} / N_{S}$ be the two partially diluted margins. We assume that the ballot manifest and the CVR both indicate that $N$ ballots were cast overall, and that ballot cards are where the manifest says they are: There are no "phantoms" in the terminology of Bañuelos and Stark [3]. We find sample sizes for a risk limit of 0.05 on the assumption that the rate of one-vote overstatements will be 0.001 . We assume that other types of errors did not occur.

Absent CSD, the sample for auditing contest $S$ would be drawn from the entire population of $N$ ballots. Contest $S$ is on $p N$ cards, so, in addition to the cards that contain undervotes or invalid votes in contest $S$, there are in effect another $(1-p) N$ cards with non-votes in contest $S$. The fully diluted margin for contest $S$ is thus $M_{S} / N=p m_{S}$. For simplicity, we use the same risk limit for both contests (5\% in our numerical examples). The number of cards we need to sample will be the smaller of the sample size for contest $B$ (with fully diluted margin $m_{B}$ and risk limit $\alpha$ ), and for contest $S$ (with fully diluted margin $p m_{S}$ and risk limit $\alpha$ ). If $m_{B} \leq p m_{S}$, then we will be done auditing both contests when the audit of contest $B$ is complete (assuming the number of discrepancies was not larger than anticipated). However, if $m_{B}>p m_{S}$ we must sample more ballots to finish the audit of contest $S$.

Here's an example. Suppose $N=10,000, p=0.1$, and $m_{B}=0.1=m_{S}$. For contest $B$, there are 5,500 reported votes for the winner and 4,500 reported votes for the loser; for contest $S$ there are 550 votes for the winner and 450 votes for the loser. Contest $B$ has an initial sample size of 64 cards, and contest $S$ has an initial sample size of 721 cards. Therefore, we will need to sample 721 cards. In general, for contest $S$, we would need to sample approximately $1 / p$ times more cards than if we sampled just from the cards that contain contest $S$. When $m_{S}$ and $p$ are small, this can lead to very large samples. For example, if $p=0.01$ and $n$ is the number of cards we would need to inspect if we were sampling just from cards that contained the contest with partially diluted margin $m_{S}$, then we could need to sample approximately $100 \mathrm{n}$ cards if we sampled from all cards (not just those that contain contest $S$ ).

If there are CSD, then the sample can be substantially smaller: We could first sample from all the cards until the audit of contest $B$ is complete. Then we can use CSD to draw additional cards that contain contest $S$.

Consider the same example as before with $N=10,000, p=0.1$, and $m_{B}=0.1=m_{S}$, but suppose we have CSD. To audit $B$ we still need to draw 64 cards. We expect that $64 p=6.4 \approx 6$ of those cards will also contain contest $S$ and $64(1-p) \approx 58$ will not. To finish the audit of $S$, we expect to need to draw 58 more cards, all containing contest $S$, for a total of 122 cards. CSD reduce the number of audited cards from 721 to about 122, a factor of almost 6 .

Figure 3 displays total ballot cards needed to audit contests $B$ and $S$, with and without CSD, across a range of partially diluted margins $m_{B}=m_{S}$ and proportions $p$ of cards on which contest $S$ appears.

\footnotetext{
${ }^{6}$ The fraction $p$ can be quite small in real elections. For instance, a 2018 ballot measure in San Mateo County, California, had 687 eligible voters out of 399,591 registered voters, i.e., $p \approx 0.0017$.
} 


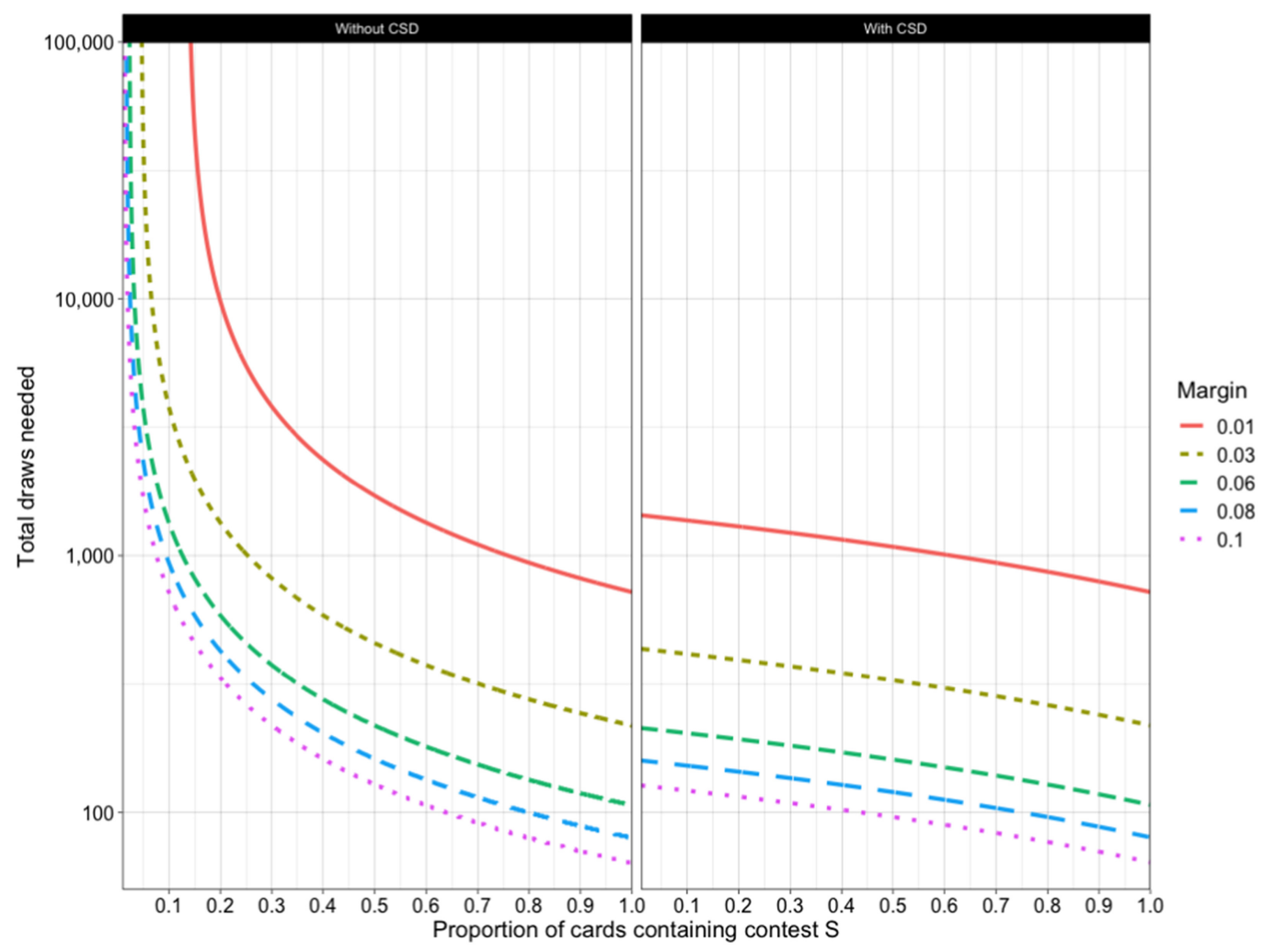

Fig. 3. Expected draws needed to confirm the outcomes in both contest $B$ and contest $S$ when the reported outcomes are correct with partially diluted margin $m_{B}=m_{S}$ given in the legend. An error rate of 0.0011 -vote overstatements is assumed. The $y$-axis is the expected number of draws (i.e., the sample size) needed on the $\log _{10}$ scale and is truncated at 100,000 draws. The left panel gives the expected number of draws if CSD are unavailable, while the right panel gives the expected number of draws with CSD. The $x$-axis ranges over a grid of proportions $p$ of ballot cards containing the small contest, from 1 in 100 ( $p=$ $0.01)$ to every ballot card $(p=1.0)$. When contest $S$ is on every card $(p=1)$, the workload is the same with or without CSD.

Figure 4 plots the number of cards needed without CSD as a percentage of the number needed with CSD. Without CSD we need to inspect substantially more ballots when $p$ is small. For very small $p$, an RLA using S4 is inefficient: A full hand count will be less work than sampling.

Figure 5 is similar to Figure 4 except $m_{S}$ is set to 0.1 and $m_{B}$ varies from 0.01 to 0.1 . When $p m_{S}>m_{B}$, there is no penalty for not having CSD.

Recall that the number of cards that must be audited is $\rho$ divided by the fully diluted margin, so the audit of contest $B$ will examine $\frac{\rho}{m_{B}}$ cards and the audit of contest $S$ will (on average) examine $\frac{\rho}{m_{S}}-\frac{p \rho}{m_{B}}$ additional cards, for a total of $(1-p) \frac{\rho}{m_{B}}+\frac{\rho}{m_{S}}$ cards. However, without CSD we would have had to examine $\frac{\rho}{p m_{S}}$ ballots. If $m_{B} \geq p m_{S}$, then CSD reduces the workload by

$$
\frac{\rho}{p m_{S}}-\left((1-p) \frac{\rho}{m_{B}}+\frac{\rho}{m_{S}}\right)
$$

on average. ${ }^{7}$

\footnotetext{
${ }^{7}$ The sample size for auditing contest $B$ is fixed, as is the sample size for auditing contest $S$, but the overlap of the two samples is random. The expected overlap is $p \rho / m_{b}$.
} 


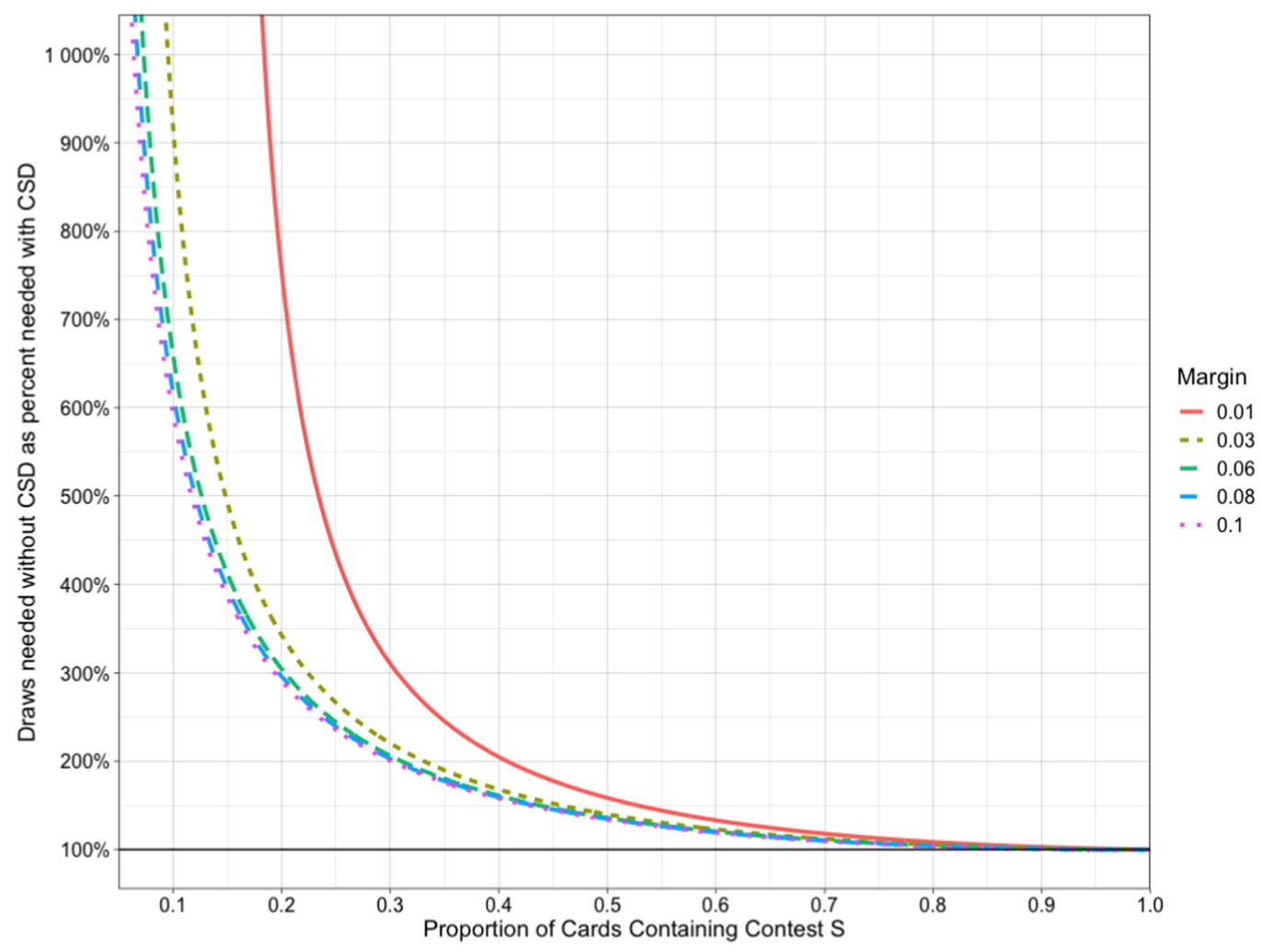

Fig. 4. Percentage increase in the number of draws required for an RLA without CSD compared to an RLA with CSD. Partially diluted margins with $m_{B}=m_{S}$ are given in the legend. Risk limit is 5\%; the audit method is S4. The $y$-axis shows expected draws without CSD as a multiple of the draws needed with CSD. For example, for a partially diluted margin of 0.03 , an audit without CSD will require inspecting about 5 times as many ballots as an audit that uses CSD if the small contest is on $15 \%$ of the ballot cards. The $y$-axis is truncated at $1,000 \%$. The $x$-axis ranges over a grid of proportions $p$ of ballot cards containing the small contest, from 1 in $10(p=0.1)$ to every ballot card $(p=1.0)$.

\section{MULTI-CARD BALLOTS}

Now suppose that each ballot consists of $c>1$ cards. For simplicity, suppose that every voter casts all $c$ cards of their ballot. Contest $B$ is on all $N$ ballots and on $N$ of the $N c$ cards. Contest $S$ is on $N p$ of the $N c$ cards.

Suppose there are $N=10,000$ ballots, $p=0.1$, and $m_{B}=0.1=m_{S}$, the risk limit $\alpha=0.05$, and we assume that the sample will reveal 1-vote overstatement errors at a rate of 0.001 , as before. Recall that for $c=1$, we had to sample 721 cards without CSD and 122 with CSD.

For $c=2$, without CSD the fully diluted margins are

$$
\frac{M_{B}}{c N}=\frac{1}{c} m_{B}=0.1 / 2=0.05
$$

for contest $B$ and

$$
\frac{M_{S}}{c N}=\frac{p}{c} m_{s}=0.1 \times 0.1 / 2=0.005
$$

for contest $S$, so the audit will examine $\rho / 0.005=1,712$ cards. 


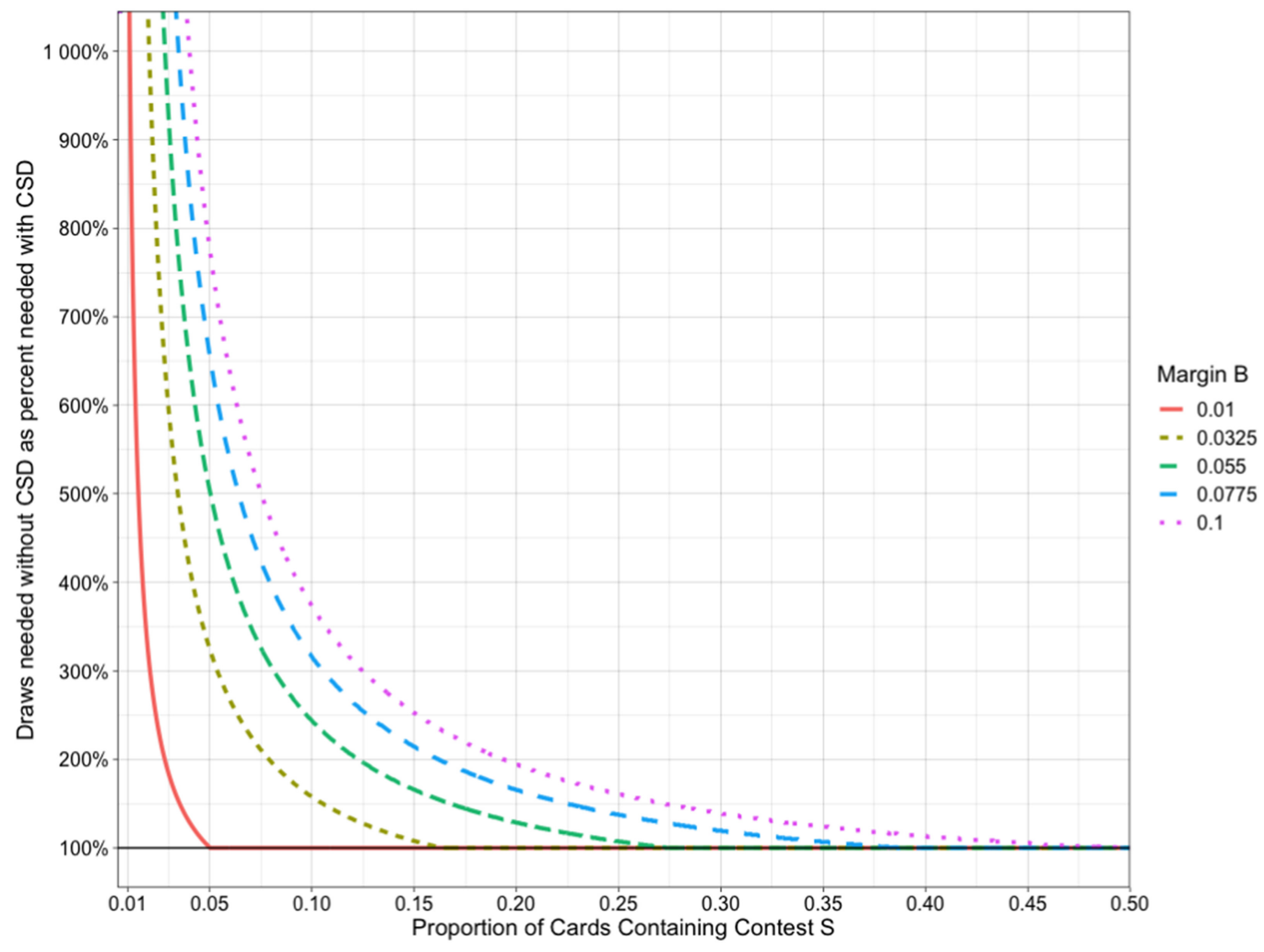

Fig. 5. Draws needed to confirm the outcomes in both contest $B$ and contest $S$ when the reported outcomes are correct, as a function of the partially diluted margin in contest $B$. Results are for the S4 auditing method and a risk limit of $5 \%$, on the assumption that the rate of 1 -vote overstatement errors is 0.001 . The partially diluted margin for contest $S$ is fixed at 0.2 . The $y$-axis gives the expected number of draws that are needed when we do not have CSD as a multiple of the number of draws needed when we do. For example, for a partially diluted margin of 0.055 , when the smaller contest is on $5 \%$ of the ballot cards, 5 times as many draws are needed if we do not have CSD. The $y$-axis is truncated at $1,000 \%$. The $x$-axis ranges over a grid of proportions $p$ of ballot cards containing contest $S$, from 1 in 100 to 1 in 2 .

For $c=5$, the fully diluted margins become

$$
\frac{1}{c} m_{B}=0.1 / 5=0.02
$$

and

$$
\frac{p}{c} m_{S}=0.1 \times 0.1 / 5=0.002
$$

without CSD, so the audit will examine 9,775 cards.

If we had CSD, then we would only need to sample 122 cards as before, no matter how large $c$ is, if every card that contains contest $S$ also contains $B$. If contests $B$ and $S$ are on different cards, then with CSD we would need to sample $64+64=128$ ballot cards, because no card that contains $S$ also contains $B$.

As the number $c$ of cards per ballot increases, the sample size without CSD grows in proportion, but the sample size with CSD stays constant: Having CSD saves more work the more cards per ballot there are. 
If we do not have CSD, then we need to examine

$$
\max \left(\frac{c \rho}{m_{B}}, \frac{c \rho}{p m_{S}}\right)
$$

cards. Suppose $m_{B}>p m_{S}$, so we need to examine $\frac{c \rho}{p m_{S}}$ ballot cards if we do not have CSD. With CSD the audit of contest $B$ will examine $\frac{\rho}{m_{B}}$ cards and the audit of contest $S$ will examine either an additional $\frac{\rho}{m_{S}}$ ballot cards if it is on a different ballot card from contest $B$ or $\frac{\rho}{m_{S}}-p \frac{\rho}{m_{B}}$ (on average) cards if every card that contains $S$ also contains $B$. This results in the following expression for the difference in the number of draws with and without CSD:

$$
\begin{array}{r}
\left(\frac{c \rho}{p m_{S}}\right)-\left(\frac{\rho}{m_{B}}+\frac{\rho}{m_{S}}\right) \text { if contests } B \text { and } S \text { are on different cards. } \\
\left(\frac{c \rho}{p m_{S}}\right)-\left((1-p) \frac{\rho}{m_{B}}+\frac{\rho}{m_{S}}\right) \text { if contests } B \text { and } S \text { are on the same cards. }
\end{array}
$$

Figure 6 plots the sample size needed without CSD as the percentage needed with CSD in an election with a multi-card ballot. The number of cards per ballot, $c$, ranges from 1 to 5, while the partially diluted margins are fixed at $m_{b}=m_{S}$. Contests $B$ and $S$ appear on the same card.

Figure 7 plots the sample size needed under the same set-up as Figure 6, except that $B$ and $S$ appear on different cards.

\section{BALLOT-POLLING AUDITS}

We have focused on comparison audits, because we expect CSD will be derived from CVRs; when the voting system can produce CVRs linked to physical ballot cards, ballot-level comparison audits are possible and save a substantial amount of work compared to ballot-polling audits.

Jurisdictions that cannot conduct ballot-level comparison audits can still conduct ballot-polling audits. We show here that CSD (derived, for instance, by physically sorting ballot cards) can yield similar savings for ballotpolling audits. Whether the savings in audit effort is worth the effort of sorting the cards will depend on the jurisdiction's logistics, details of the contests under audit (including the partially diluted margins), and the number of cards per ballot.

For ballot-polling audits, the expected sample size scales like the square of the reciprocal of the margin and linearly in the reciprocal of the fraction of ballot cards that contain the contest. In other words, without CSD the sample sizes expected for ballot-polling audits scale linearly in $1 / p$, the proportion of cards containing the smaller contest, and linearly in $c$, the number of cards per ballot-just as comparison audits do.

Consider again auditing contests $B$ and $S$ with margins $M_{B}$ and $M_{S}$ (in votes), $N$ ballots cast each consisting of $c$ cards, contest $B$ on $N$ of the $N c$ cards and $S$ is on $p N$ of the cards. For simplicity, assume that the contests are two-candidate plurality contests with no invalid votes; with small changes to the notation, the result can be generalized.

For the BRAVO method for ballot polling [11], the expected sample size is approximately $\frac{2 \ln (1 / \alpha)}{m^{2}}$ where $m$ is the margin as a fraction (the margin in votes, divided by the number of votes cast for the winner or the loser).

Suppose we know which ballots contain $S$ but not which particular cards contain $S$, and that the $c$ cards comprising each ballot are kept in the same container. This is an idealization of precinct-based voting where each voter in a precinct gets the same ballot style and casts all $c$ cards of the ballot. If voters in that precinct are eligible to vote in $S$, then a fraction $1 / c$ of the cards in the container will have contest $S$; otherwise, none of the cards in the container will have $S$.

This lets us target the sampling for auditing contest $S$, but only partially: we can reduce the sampling universe for contest $S$ from the original population of $N c$ cards to a smaller population of $p N c$ cards, of which $p N$ actually 


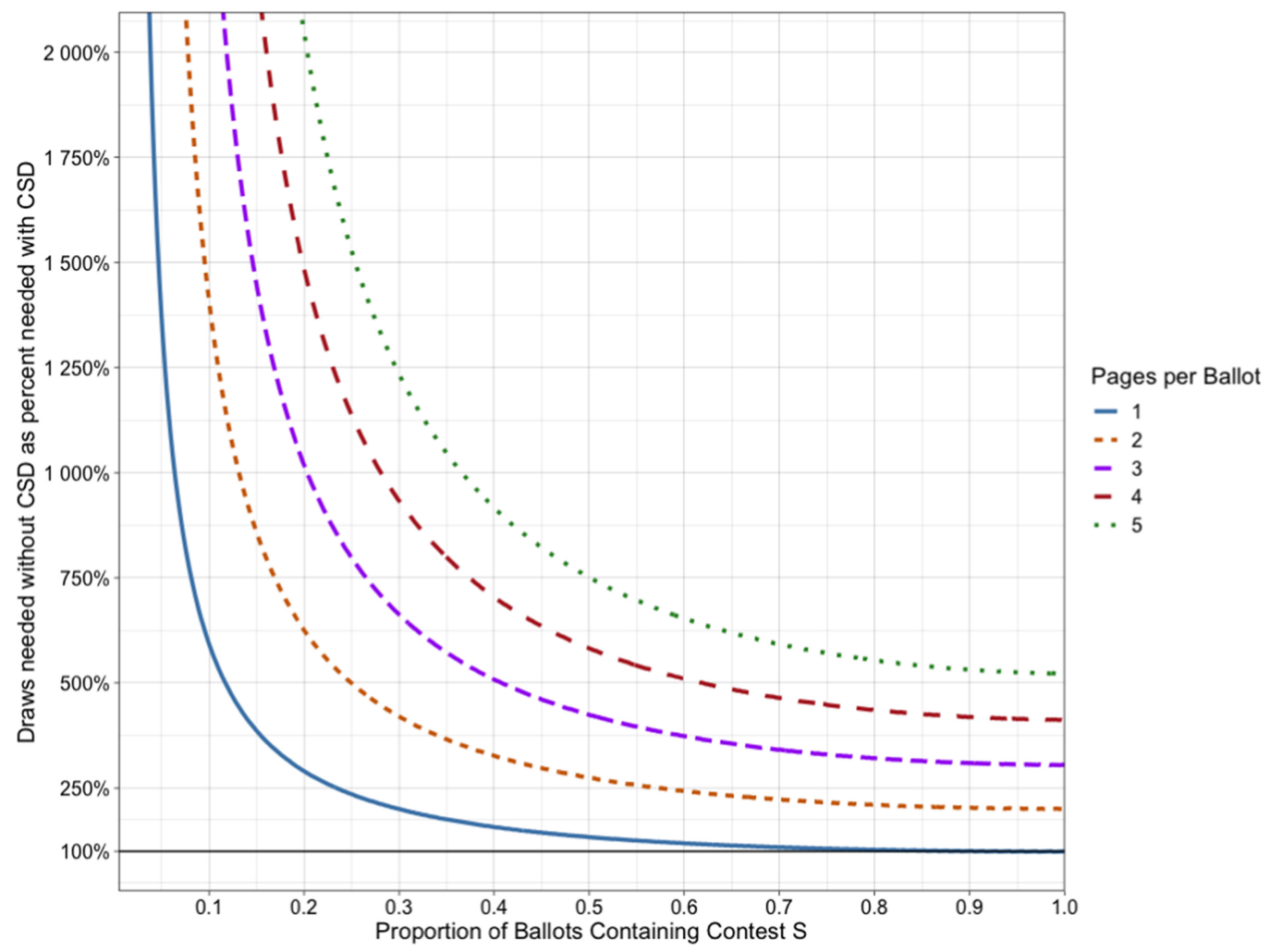

Fig. 6. Draws needed to confirm the outcome in both contests ( $B$ and $S$ ) using the $S 4$ method at risk limit $\alpha=0.05$ without CSD as a multiple of the number of draws needed with CSD ( $y$-axis), truncated at 2,000\%. The contests appear on the same ballot-card and both partially diluted margins are fixed at 0.1 . The lines indicate multiple needed; different lines correspond to different numbers of cards per ballot. The $x$-axis ranges over the proportion of ballots (not ballot cards) containing contest $S$, from 1 in $100(p=0.01)$ to all ballots $(p=1.0)$.

contain contest $S$. The sample for $B$ would be drawn from all $N c$ cards, of which $N$ contain contest $B$. The difference in expected sample sizes compared to "blind" ballot polling with no CSD is

$$
\begin{array}{r}
2 c \ln (1 / \alpha)\left(\frac{1}{p m_{S}^{2}}-\frac{1}{m_{B}^{2}}-\frac{1}{m_{S}^{2}}\right) \text { if contests } B \text { and } S \text { are on different cards. } \\
2 c \ln (1 / \alpha)\left(\frac{1}{p m_{S}^{2}}-(1-p) \frac{1}{m_{B}^{2}}-\frac{1}{m_{S}^{2}}\right) \text { if contests } B \text { and } S \text { are on the same card. }
\end{array}
$$

For a risk limit of $5 \%$ and a margin of $10 \%$, using BRAVO, we would expect to sample 608 cards if we could target the sample. Consider the example $N=10,000, p=0.3, M_{B}=0.1=M_{S}$. If $c=2$, then absent CSD we would expect to sample $(2 / 0.3) \times 608=4,053$ cards. With partial CSD, but no information about which contests are contained on an individual card, we would expect to sample $(1-0.3) 2 \times 608+2 \times 608=2,067$ cards if the contests are on the same card and $2 \times 608+2 \times 608=2,432$ ballot cards if the contests are on different cards. In either case, using CSD reduces the sample size by roughly half.

Thus, information about which containers have which card styles-even without information about which cards contain which contests-can still yield substantial efficiency gains for ballot-polling audits. 


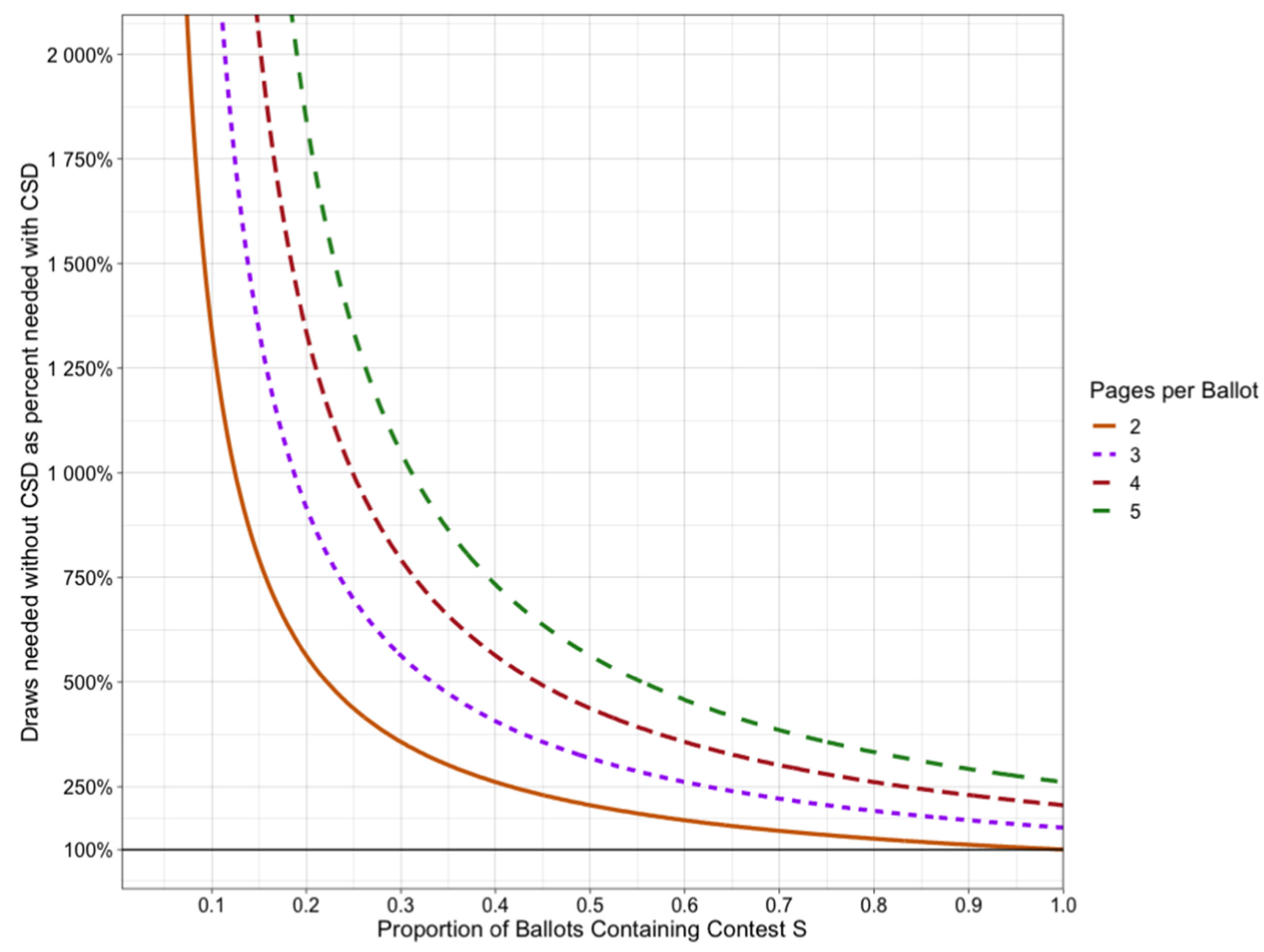

Fig. 7. Draws needed to confirm outcome in both contests ( $B$ and $S$ ) when we do not have CSD expressed as a percentage of draws needed with CSD ( $y$-axis), truncated at 2,000\%. The contests appear on different pages, while both partially diluted margins are fixed at 0.1 . The lines indicate percentage of draws needed, colored by the number of pages in the ballot. The $x$ axis ranges over the proportion of ballots (not ballot cards) containing contest $S$, from 1 in $100(p=0.01)$ to all ballots $(p=1.0)$.

\section{CASE STUDIES}

We will give numerical examples for Inyo County, California (a small county), and Orange County, California (a large county), both of which have conducted several RLA pilots or binding RLAs.

\subsection{Inyo County, California}

Inyo County (population 18,546 according to the 2010 U.S. Census) is in eastern California. The county conducted a pilot RLA of an April 2018 special election using the S4 method [8] (it has conducted other RLAs as well, including RLAs of seven contests in the 2020 general election).

In the June 2018 election each ballot consisted of two cards $(c=2)$. We consider two contests on this ballot: Supervisor District 1 (contest $S$ ) and U.S. Senator (contest $B$ ). (We shall pretend that the Senate contest was entirely contained in Inyo County.) Of the 5,919 ballots cast in the June 2018 election, 1,435 ( $p=0.24)$ contained the Supervisor District 1 contest. This contest had a partially diluted margin of $m_{S}=36 / 1,435=0.025$. In California primaries, the top two candidates with the most votes (from either party) advance to the general election, so the margin between the second place candidate and the third place candidate drives the audit effort. All voters in Inyo County were eligible to vote in the U.S. Senate contest. Diane Feinstein received the most votes, 1,555, followed by James Bradley with 639, and Paul Taylor with 517. We consider confirming that Feinstein and 
Table 1. Expected Percentage Reduction in the Sample Size Required with CSD versus without CSD to Audit the Contests Described in Section 6

\begin{tabular}{|l|c|c|}
\hline & Comparison Audit & Ballot-Polling Audit \\
\hline Inyo County & $84 \%$ & $29 \%$ \\
\hline Orange County & $85 \%$ & $67 \%$ \\
\hline
\end{tabular}

Bradley received more votes than Taylor. The partially diluted margin for this contest is

$$
m_{B}=(639-517) / 5,919=0.02>0.006=p m_{S} .
$$

First, consider a comparison audit. Without CSD, we would expect to sample 3,734 cards. The Supervisor District 1 and U.S. Senate contests were not on the same card, so with CSD we would expect to sample 588 cards, smaller by about $3,734-588=3,146$ cards than an audit without CSD.

Suppose instead we audit using BRAVO, a ballot-polling method. Without CSD, we would expect to audit $2,796 \times 2$ ballot cards to verify contest $B$, but to confirm the outcome of contest $S$ would essentially require a full hand count of the votes on all 11,838 cards. If we knew which containers had cards that include contest $S$, then we would expect to audit $2,796 \times 2+1,435 \times 2=8,462$ cards. (While this is a substantial reduction, it is probably more efficient to conduct a full hand count than to examine a majority of ballot cards selected randomly.)

\subsection{Orange County, California}

Orange County, in Southern California, is much larger (3.017 million residents according to the 2010 U.S. Census). The ballots in their 2018 election consisted of $c=2$ cards. A BRAVO RLA of three of the five countywide contests in 2018 was conducted [15].

Suppose we wanted to audit the Senate (Feinstein v De Leon) and 45th District Congressional (Porter v Walters) contests in Orange County from the November 2018 election. (As before, we shall pretend for the purpose of illustration that the senatorial contest is entirely contained in Orange County.) All voters were eligible to vote in the Senate contest. Of the 1,106,729 ballots cast, 312,700 (28.25\%) included the 45th District Congressional contest. In this case $p \approx 0.2825, m_{S}=0.04, m_{B}=0.073$, and $c=2$, so

$$
m_{B}=0.073>0.012=p m_{S} .
$$

First consider a ballot-level comparison audit. Without CSD, we would expect to have to sample 1,452 cards. With CSD, we would expect to have to sample 249 cards if the contests were on different cards, and 225 if they were on the same card. These two contests were in fact on the same ballot card, so using CSD would decrease the expected number of audited cards by $1,452-225=1,227$.

Suppose instead we audit using the BRAVO ballot-polling method. If we did not have CSD, then we would expect to have to sample $2 * 3,671 / 0.2825=25,989$ ballot cards. If ballots were organized by ballot style (but not card style), then we would expect to sample $(1-0.2825) \times 2 \times 948+2 \times 3,671=8,702$ cards.

Table 1 summarizes the results of this section, the expected percentage reduction in number of ballot cards drawn with CSD versus without CSD.

\section{IMPLEMENTATION}

To minimize the number of cards a risk-limiting audit of multiple contests needs to inspect, we would like to be able to use any card in the audit sample to audit every contest that card contains. Standard methods for ballotlevel comparison audits or ballot-polling audits then require that the intersection of the sample with the cards that contain each contest is a uniform random sample from that contest (either with or without replacement, as 
a)

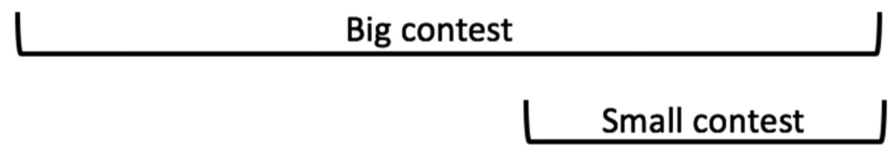

b)

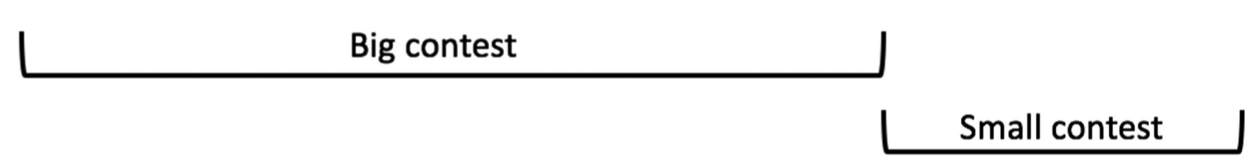

c)

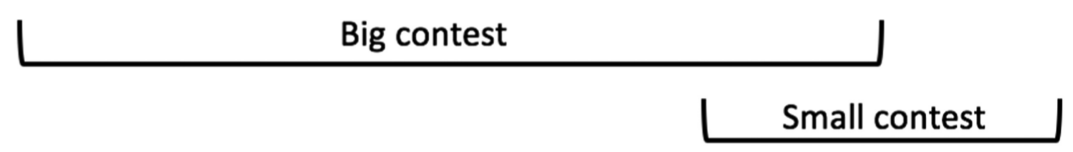

Fig. 8. Overlap of card styles in three hypothetical elections with two contests (one big and one small). Case $a$ represents complete nesting, where uniform draws from the big contest yield uniform draws from the small contest. If all ballots consist of a single ballot card and the big contest is on all ballots, then the situation is case $a$. Case $b$ represents a scenario where ballot cards that contain the big contest do not contain the small contest. Auditing the big contest tells us nothing about the small contest. In case $c$, the small contest sometimes appears on the card for the big contest and sometimes not. Drawing ballot cards uniformly from the big contest does not sample from the entire small contest.

appropriate for the auditing method). When the contests are on some of the same cards, this requires particular care. Figure 8 sketches the possibilities.

One method for ensuring uniformity on overlapping subsets is called consistent sampling. Consistent sampling can be done with replacement [14] or without replacement [6, 7]. It is simpler without replacement, which also leads to more efficient RLAs [19]. Figure 9 illustrates consistent sampling with a toy example of auditing two contests in a small election.

Here we show how to audit $K$ contests of different sizes using CSD and consistent sampling. We assume that (1) there has been a compliance audit to establish that the paper trail is trustworthy $[2,4,18,20] ;$ (2) there is an upper bound on the number of cards that contain each contest under audit, obtained, for example, from pollbook signatures and other administrative records; and (3) the underlying auditing method (comparison vs ballot-polling, and the "risk function"), sampling method, and risk limits $\left(\left\{\alpha_{1}, \ldots \alpha_{K}\right\}\right)$ have been chosen, along with rules for picking the initial sample size(s) $\left\{S_{1}, \ldots, S_{K}\right\}$ and for increasing the sample size if the audit does not confirm the outcome with the initial sample.

(1) If there are more CVRs that contain any contest than the upper bound on the number of cards that contain the contest, then stop: The contest outcome cannot be confirmed.

(2) If the upper bound on the number of cards that contain a contest is greater than the number of CVRs that contain the contest, then create a corresponding set of "phantom" CVRs as described in Section 3.4 of Stark [19].

(3) If the upper bound on the number of cards that contain a contest is greater than the number of physical cards whose locations are known, then create enough "phantom" cards to make up the difference.

(4) Assign a $U[0,1]$ pseudo-random number to every ballot card that contains one or more contests under audit (including "phantom" cards), using a high-quality PRNG [12]. For sampling without replacement, assign one number to each card; for sampling with replacement, assign several, as described in Rivest [14]. 


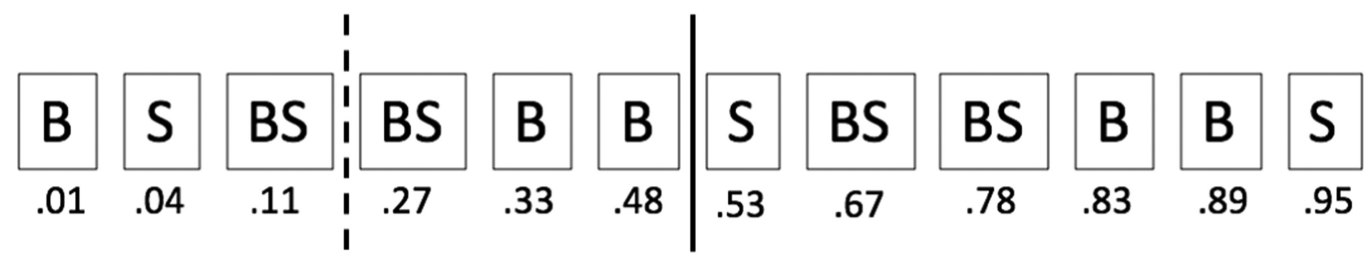

Fig. 9. Illustration of consistent random sampling without replacement to audit 2 contests, $B$ and $S$. Contest $B$ appears on 9 cards and $S$ appears on 7 cards; 12 cards were cast in all. There is partial overlap (case c of Figure 8): Contest $B$ appears alone on 5 cards (labelled "B"), the contests appear together on 4 cards (labelled "BS"), and contest $S$ appears alone on 3 cards (labelled " $\mathrm{S}$ "). To perform consistent sampling, each card is assigned a random number in $[0,1]$ independently across cards. The number appears below each card; without loss of generality, we have sorted the cards by increasing order of the random number. Suppose the audit requires inspecting 5 cards containing $B$ and 2 cards containing $S$. The 5 sampled cards containing $B$ are the cards containing $B$ that were assigned the 5 smallest numbers, i.e., all cards containing $B$ to the left of the second (solid) vertical bar. The 2 sampled cards containing $S$ are the cards containing $S$ that were assigned the 2 smallest random numbers, i.e., all cards containing $S$ to the left of the first (dashed) vertical bar. This approach ensures that if we look at a card to audit one contest and the other contest is also on the card, it can be used in the audit of that contest, too: the sample is uniformly distributed on subsets of the population. This approach can be generalized to sampling with replacement; see Rivest [14].

(5) Initialize $\mathcal{A}$ to be the set of contests under audit: $\mathcal{A} \leftarrow\{1, \ldots, K\}$.

(6) While $\mathcal{A}$ is not empty:

(a) Pick the sample sizes $\left\{S_{k}\right\}$ for $k \in \mathcal{A}$ for this round of sampling.

(b) Choose thresholds $\left\{t_{k}\right\}_{k \in \mathcal{A}}$ so that $S_{k}$ ballot cards containing contest $k$ have numbers less than or equal to $t_{k}$.

(c) Retrieve any of the corresponding ballot cards that have not yet been audited and inspect them manually. If there is no CVR for the ballot, then treat the CVR as if it recorded a non-vote in every contest still under audit. If a ballot card cannot be found or if it is a phantom card, then treat it in the way that casts the most doubt on the outcome of every audited contest it was supposed to contain (see Section 3.4 of Stark [19]). ${ }^{8}$

(d) Use the data from the previous step to update the measured risk for every contest $k \in \mathcal{A}$.

(e) Remove from $\mathcal{A}$ all $k$ that have met their risk limits.

\section{CONCLUSIONS}

CSD can dramatically increase the efficiency of risk-limiting audits. For the super-simple simultaneous singleballot audit of Stark [17] and for ballot-polling audits using BRAVO [11], the expected reduction in sample size can easily be several orders of magnitude, depending on the range of sizes and margins of the contests under audit and the number of cards per ballot.

When a contest is on only a small fraction of the cards cast in an election and the sample is drawn from all cast cards, auditing the contest can be nearly as much work as a full manual tally involving all cast cards, not just those that contain the contest-even if the margin in the contest is large. In contrast, if there are CSD so that the sample can be drawn from just those cards that (reportedly) contain the contest, auditing small contests can be quite efficient.

Jurisdictions that perform RLAs should consider using CSD to reduce the workload of auditing small contests and contests on multi-card ballots: the savings can be substantial. Jurisdictions that can conduct ballot-level comparison audits (i.e., jurisdictions with voting systems that can export CVRs linked to physical ballots) can

\footnotetext{
${ }^{8}$ Some cards may be selected for auditing more than one contest. If the sample is drawn with replacement, then the same card may be selected more than once. Such cards are only manually inspected once, even though their data might be re-used.
} 
construct CSD with no additional effort, because the CVRs contain CSD-albeit, possibly erroneous. However, to ensure that errors in the CVRs resulting in errors in CSD do not compromise the risk limit, jurisdictions also need an upper bound on the total number of ballot cards that contain each contest, information that can be derived from pollbooks and related administrative voter records.

\section{ACKNOWLEDGMENTS}

Thank you to Kammi Foote and Neal Kelley for providing data for the Inyo and Orange County case studies. Thanks also to the anonymous reviewers for their helpful feedback.

\section{REFERENCES}

[1] A. W. Appel, R. DeMillo, and P. B. Stark. 2020. Ballot-marking devices cannot assure the will of the voters. Elect. Law f. 19, 3 (2020), $432-450$.

[2] A. W. Appel and P. B. Stark. 2020. Evidence-based elections: Create a meaningful paper trail, then audit. Georgetown Law Technol. Rev. 4, 2 (2020), 523-541.

[3] J. H. Bañuelos and P. B. Stark. 2012. Limiting Risk by Turning Manifest Phantoms into Evil Zombies. Technical Report.

[4] J. Benaloh, D. Jones, E. Lazarus, M. Lindeman, and P. B. Stark. 2011. SOBA: Secrecy-preserving observable ballot-level audits. In Proceedings of the 2011 Electronic Voting Technology Workshop/Workshop on Trustworthy Elections (EVT/WOTE'11). USENIX, California, 13.

[5] M. Blom, A. Conway, D. King, L. Sandrolini, P. B. Stark, P. J. Stuckey, and V. Teague. 2020. You can do RLAs for IRV. In Proceedings of the International Conference for Electronic Voting (E-VOTE-ID’20), R. Krimmer, M. Volkamer, B. Beckert, A. D. Maurer, D. Duenas-Cid, S. Glondu, I. Krivonosova, O. Kulyk, R. Küsters, B. Martin-Rozumilowicz, P. Ronne, M. Solvak, and O. Spycher (Eds.). TalTech Press, Tallinn, Estonia, 296-310.

[6] A. Broder. 1997. On the resemblance and containment of documents. In Compression and Complexity of Sequences. IEEE, $21-29$.

[7] A. Broder, S. Glassman, M. Manasse, and G. Zweig. 1997. Syntactic clustering of the web. In Selected Papers from the 6th International World Wide Web Conference. Elsevier Science Publishes Ltd., Essex, UK, 1157-1166.

[8] K. Foote. 2018. Report on the Inyo County Risk-Limiting Audit Pilot. Retrieved November 30, 2020 from https://lhc.ca.gov/sites/lhc.ca. gov/files/Reports/247/WrittenTestimony/FooteJuly2018.pdf.

[9] M. Lindeman, N. McBurnett, K. Ottoboni, and P.B. Stark. 2018. Next steps for the Colorado Risk-Limiting Audit (CORLA) program. arXiv:1803.00698. Retrieved from https://arxiv.org/abs/1803.00698.

[10] M. Lindeman and P. B. Stark. 2012. A gentle introduction to risk-limiting audits. IEEE Secur. Priv. 10 (2012), $42-49$.

[11] M. Lindeman, P. B. Stark, and V. Yates. 2012. BRAVO: Ballot-polling risk-limiting audits to verify outcomes. In Proceedings of the 2011 Electronic Voting Technology Workshop / Workshop on Trustworthy Elections (EVT/WOTE'11). USENIX, 12.

[12] K. Ottoboni and P. B. Stark. 2019. Election integrity and electronic voting machines in 2018 georgia, USA. In Proceedings of the International Conference for Electronic Voting (E-Vote-ID'19), Lecture Notes in Computer Science. Springer Nature, Switzerland, 166-182.

[13] K. Ottoboni, P. B. Stark, M. Lindeman, and N. McBurnett. 2018. Risk-limiting audits by stratified union-intersection tests of elections (SUITE). In Proceedings of the International Conference for Electronic Voting (E-Vote-ID'18) Lecture Notes in Computer Science. Springer, Switzerland.

[14] R. L. Rivest. 2018. Consistent sampling with replacement. arXiv:1808.10016v1. Retrieved from https://arxiv.org/abs/1808.10016v1.

[15] S. Singer and N. McBurnett. 2018. Orange County, CA pilot risk-limiting audit. Retrieved November 30, 2020 from https://www. verifiedvoting.org/wp-content/uploads/2018/12/2018-RLA-Report-Orange-County-CA.pdf.

[16] P. B. Stark. 2008. Conservative statistical post-election audits. Ann. Appl. Stat. 2 (2008), 550-581.

[17] P. B. Stark. 2010. Super-simple simultaneous single-ballot risk-limiting audits. In Proceedings of the 2010 Electronic Voting Technology Workshop / Workshop on Trustworthy Elections (EVT/WOTE'10). USENIX Association, Berkeley, CA, 1-16.

[18] P. B. Stark. 2018. An Introduction to Risk-Limiting Audits and Evidence-Based Elections, Written Testimony Prepared for the Little Hoover Commission. Retrieved from https://www.stat.berkeley.edu/ stark/Preprints/lhc18.pdf.

[19] P. B. Stark. 2020. Sets of half-average nulls generate risk-limiting audits: SHANGRLA. In Proceedings of the International Conference on Financial Cryptography and Data Security, R. Krimmer, M. Volkamer, B. Beckert, A. D. Maurer, D. Duenas-Cid, S. Glondu, I. Krivonosova, O. Kulyk, R. Küsters, B. Martin-Rozumilowicz, P. Ronne, M. Solvak, and O. Spycher (Eds.). Springer, Cham, $319-336$.

[20] P. B. Stark and D. A. Wagner. 2012. Evidence-based elections. IEEE Secur. Priv. 10 (2012), 33-41.

Received May 2020; revised December 2020; accepted March 2021 